\title{
Effect of Voltage Constraints on the Exchange of Flexibility Services in Distribution Networks
}

\author{
Sébastien Mathieu, Frédéric Olivier, Damien Ernst, Bertrand Cornélusse \\ Department of Electrical Engineering and Computer Science \\ University of Liège, Belgium \\ \{sebastien.mathieu, frederic.olivier, dernst, bertrand.cornelusse\}@ulg.ac.be
}

\begin{abstract}
Many possibilities exist to organise exchanges of flexibility within a distribution system. In this paper, we call such a possibility an interaction model. The DSIMA (Distribution System Interaction Model Analysis) testbed allows one to compare quantitatively candidate interaction models by simulating a distribution system with actors taking decisions to maximise their own profit or minimise their costs. The original testbed focused on establishing the procedures to exchange information between actors and used a network flow model considering only active power. This paper extends DSIMA with a linear approximation of the power flow equations, the line limits and the voltage constraints. This linear flow model is compared to a network flow model by simulating three different interaction models governing the exchange of flexibility services within a Belgian distribution system. Results show that changing the network model may significantly impact the quantitative results obtained from the simulations.
\end{abstract}

\section{INTRODUCTION}

Distribution systems were typically sized to supply the empirically observed peak consumption, and to require few preventive or corrective control actions, by making the assumptions that the grid users were only consuming power in a non-coordinated way. However, the development of distributed generation, renewable or not, and demand-side management is likely to lead to many issues in operation by making those assumptions less realistic. Two main options are available to overcome these issues: network reinforcement and active network management [1]. The latter involves increasing the efficiency of distribution systems operating the system using all control means available, and the flexibility of the grid users, which can be offered as flexibility services taking the form of modulations with respect to their intended production or consumption.

This change of practices in distribution systems calls for a revision of the interaction model, that is, the set of rules guiding the interactions between all the parties of the system. A new interaction model is usually chosen after a discussion between affected actors, based on qualitative analyses making simplistic assumptions. Using only qualitative analyses can lead to unexpected behaviours of the actors and a lower social welfare. To avoid such consequences, one should rely on quantitative analyses, for example with agent-based modelling [2], to determine the financial impact of changing the interaction model. Since actors are modelled as individuals, every exchange of information and financial transaction has to be modelled explicitly. Therefore, implementing a given interaction model into an agent-based model provides insights on the difficulties to implement the interaction model from a technical perspective, and highlights the necessary exchanges of information that a qualitative analysis could have missed.

To compare alternative interaction models relying on active network management, an agent-based system has been devised and published as an open-source testbed: DSIMA (Distribution System Interaction Model Analysis) [3]. Within this framework, each agent optimises its individual objective following the rules guided by the interaction model. This article extends the original network flow model used in optimisation problems solved by the Distribution System Operator (DSO), with a flow model that considers reactive power flow and voltage constraints. In order to keep the simulation computationally tractable, the updated flow model is a linear version of the classic optimal power flow (OPF) formulation based on [4], which provides a linear relation between the voltages and the injected power at each bus, and bounds the approximation error. Simulations are performed with DSIMA to quantify the effect of the flow models under different interaction models.

The paper is organised as follows. The literature relevant to the main methodological addition to [3] is reviewed in Section II. Section III describes the original DSIMA testbed in more detail and presents the evaluated interaction models. The linear flow model and its implementationare proposed in Sections IV and V. Section VI discusses the simulation results, and Section VII concludes.

\section{LITERATURE REVIEW}

The linearised direct current (DC) power flow approximation is often used to solve large-scale systems [5] but is not well suited to represent distribution networks since the model does not accurately capture under- or over-voltages. Alternative linear models have been proposed to overcome this issue. For instance, the power flow equations can be linearly approximated by considering their perturbations with respect to deviations from references of active and reactive power injection in each bus [6]. Another linear approximation is proposed in [7] and compared to the DC power flow approximation on experimental test systems. On a more theoretical perspective, [4] provide a linear relation between the voltages and the injected power at each bus, and bound the approximation error. The latter formulation is used in our analysis to model the power flows in the distribution networks. 


\section{DEFINITION OF THE INTERACTION MODELS AND THEIR IMPLEMENTATION IN DSIMA}

This section recalls the main elements of the system modelled in the open-source testbed DSIMA, see [3] for more details. We consider the short-term flexibility exchanges in an operational planning phase of a medium-voltage network. The actors simulated are producers and retailers, which are called Grid Users (GUs), the DSO and the Transmission System Operator (TSO). A grid user is responsible of its imbalance and may use or provide flexibility services to other grid users as well as the DSO and TSO.

\section{A. Access bounds and access contracts}

We assume that each GU has an access contract with the DSO for each bus it has access to. This contract specifies a time-invariant full-access range, in which the GU can produce or consume without any restriction. Each GU first requests a specific access range to the DSO for each bus it wants to access. Based on that, the DSO computes the safe access range, which is lower or equal to the requested range and ensures that no congestion occurs if every GU accesses to the network within these limits. One method to perform this computation is explained in [3].

Three access types are considered, respectively named: restricted, unrestricted and dynamic. In the first access type, the GU production or consumption must always remain within the safe access range, i.e. its full access range is equal to the safe access range. For the second, no restrictions are applied to GUs, and the DSO relies on flexibility services to operate its network. Stakeholders are free to exchange flexibility services among them, and in particular with the DSO. For the third, the DSO can restrict the access of the GU to a dynamic range, which changes for each time period, e.g. 15 minutes, and constrained to be larger than the full access range.

\section{B. Interaction models}

The interaction models implemented in DSIMA follow the procedure below and summarised in Figure 1, where some steps may be skipped. (i) GUs provide baseline proposals. (ii) Based on these, the DSO computes the dynamic ranges so that its network is secure and communicates them to the GUs. (iii) After the clearing of the day-ahead energy market, GUs submit their (new) baselines, constrained to lie within the dynamic ranges. They are used as references for the provision of flexibility services. If the realisation of a $\mathrm{GU}$ violates the dynamic ranges, the $\mathrm{GU}$ is penalised at a regulated tariff. (iv) The DSO assesses the state of the system and they and other GU announces their flexibility needs. (v) GUs provide flexibility offers sequentially to the other actors, the first being the DSO. (vi) GUs request and/or buy some proposed flexibility offers. (vii) Closer to real-time, activation requests of flexibility services are communicated to the GUs. (viii) Right before real-time, each GU optimises its realisation, taking into account the requests. (ix) The distribution network is operated using these realisations and takes last resort actions if necessary, such as shedding buses. If such actions are needed and GUs did not provide their service to the DSO, they are penalised at a regulated price.

Given the previous definitions, this paper focuses on three interaction models:

- Model 0, where the access type is restricted, and there is no financial compensation for flexibility,

- Model 1, where the access type is dynamic, and the DSO does not use flexibility to operate its network.

- Model 2, where the access is unrestricted and the DSO pays a reservation and activation cost and pays for the resulting imbalance caused by the activation of flexibility services.

The analysis could be extended to others, such as the remaining three models presented in previous work [3], [8].

\section{LINEAR POWER FLOW}

The traditional power flow equations use the admittance of the lines to evaluate the voltages and obtain the losses. They can be written as:

$$
\begin{gathered}
\forall n \in \mathcal{N}, n^{\prime} \in \mathcal{N}(n), t \in \mathcal{T}, \\
s_{n, t}=\sum_{n^{\prime} \in \mathcal{N}(n)} S_{n, n^{\prime}, t} \\
V_{n}^{\text {min }} \leq\left\|V_{n}\right\|_{2} \leq V_{n}^{\max } \\
\bar{S}_{n, n^{\prime}, t}=\bar{V}_{n, t} Y_{n, n^{\prime}}\left(V_{n, t}-V_{n^{\prime}, t}\right) \\
\left\|S_{n, n^{\prime}, t}\right\|_{2} \leq C_{n, n^{\prime}}
\end{gathered}
$$

where $\mathcal{N}$ is the set of buses, $\mathcal{N}(n)$ the set of buses connected to bus $n, \mathcal{T}$ the set of time periods, $t$ the time period, $V_{n, t}=$ $e_{n, t}+j f_{n, t}$ the complex voltage at bus $n, S_{n, n^{\prime}, t}=p_{n, n^{\prime}, t}+$ $j q_{n, n^{\prime}, t}$ the complex power flowing from bus $n$ to bus $n^{\prime}$, and $s_{n, t}$ the complex power injection at bus $n$. The conjugate of a complex number $x$ is denoted $\bar{x}$ and its two-norm $\|x\|_{2}$. The main complexity of this formulation originates from the nonlinearity of the quadratic term in the equality constraint (1c) and the concave minimum bound in (1b).

In this study, (1c) is approximated using Bolognani and Zampieri's work [4]. They show that the voltages in each buses, in a column vector, can be approximated by

$$
\hat{\mathbf{V}}_{t} \simeq V_{0}\left(\mathbb{1}+\frac{1}{\left\|V_{0}\right\|_{2}^{2}} Z \overline{\mathbf{S}}_{t}\right)
$$

where $\mathbf{S}_{t}$ is the column vector with the complex power injections and $V_{0}$ is the complex voltage of the slack bus. The matrix $Z$ is defined as the inverse of the nodal admittance matrix and $\mathbb{1}$ is a column vector of ones. With our notations, by developing (2) and neglecting the shunt admittances, one can obtain:

$$
\bar{S}_{n, n^{\prime}, t}=\bar{V}_{0} Y_{n, n^{\prime}}\left(V_{n, t}-V_{n^{\prime}, t}\right)
$$

The necessary data are the resistance and reactance of all lines, and the active and reactive power injections at each bus. Note that this linear approximation yields $S_{n, n^{\prime}, t}=-S_{n^{\prime}, n, t}$ and therefore the power flows are conserved. As a result, no losses occur in the system and only the voltage differences are approximated. 


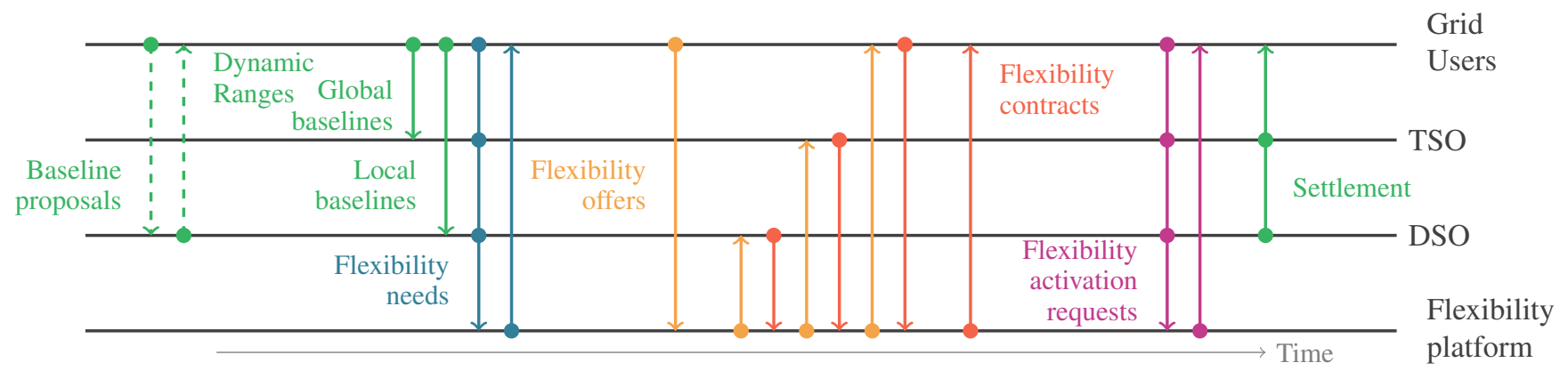

Fig. 1: Interactions between the agents where each type of actor is represented by a horizontal line. Each vertical arrow represents an interaction between two types of actor.

As shown in Figure 2, a convex circled-shape constraint can be approximated by a conservative feasible set of cuts. This approximation can be used for the constraint on the flow in each line and the constraint on the maximum voltage. If $I$ is the number of cut points by quadrant of the complex plane, $\mathcal{I}=\{1, \ldots, I\}$ and $\Phi(I)$ is an ordered set with the corresponding angles:

$$
\Phi(I)=\left\{0, \frac{\pi}{2 I}, \frac{2 \pi}{2 I}, \frac{3 \pi}{2 I}, \ldots, \frac{\pi}{2}\right\},
$$

the set of constraints defining the boundary in the north-east quadrant of the complex plane of $S_{n, n^{\prime}, t}=p_{n, n^{\prime}, t}+j q_{n, n^{\prime}, t}$ is given by, $\forall i \in \mathcal{I}$

$$
A_{i} p_{n, n^{\prime}, t}+B_{i} q_{n, n^{\prime}, t} \leq A_{i} C_{n, n^{\prime}},
$$

where $A_{i}=\sin \Phi_{i}-\sin \Phi_{i-1}, B_{i}=\cos \Phi_{i}-\cos \Phi_{i-1}$.

The constraints bounding the three other quadrants can be obtained by changing the signs of the $p_{n, n^{\prime}, t}$ and $q_{n, n^{\prime}, t}$ terms in (5). These constraints are too conservative and the maximal error margin can be easily computed by trigonometry:

$$
\frac{1}{2} \sqrt{\left(\cos \frac{\pi}{2 I}+1\right)^{2}+\sin ^{2} \frac{\pi}{2 I}}
$$

which for $I=4$ leads to a conservative error of $1.92 \%$.

The same method may be used to approximate the bound on the maximum voltage amplitude, but not the one on the minimum voltage amplitude, which is concave. This work chooses to conservatively approximate the feasible domain of the voltage with the constraint $e_{n, t} \geq V_{n}^{\text {min }}$, as shown in Figure 2. This approximation is acceptable for angle deviations that are relatively small, which is often the case in practice. Denoting $\theta_{n, t}$ the angle of the voltage of the bus $n$ where $e_{n, t}=V_{n}^{\text {min }}$, the conservative error margin is given by $1 / \cos \theta_{n, t}$ (e.g. $3.5 \%$ when $\theta_{n, t}=15^{\circ}$ ).

\section{INTEGRATION IN THE DSO OPERATIONS}

In DSIMA, the linear power flow model is used in all optimisation problems solved to define the behaviour of the DSO, from the definition of the full access ranges to the realtime operation of the network. This section describes how the testbed is updated so that the DSO uses the linear flow models.

Since a design choice of the testbed is to never obtain an infeasible optimisation problem in order to always have results

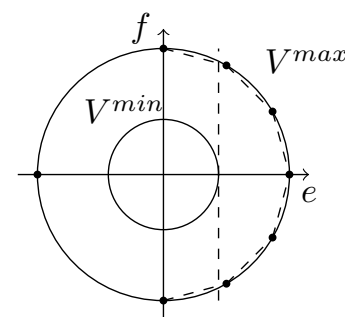

Fig. 2: Linear approximation of the constraint $V^{\text {min }} \leq \| e+$ $j f \|_{2} \leq V^{\max }$ with three cut points.

to analyse, the voltage constraints are converted into soft constraints, where $\zeta_{n, t}$ and $\nu_{n, t}$ are slack variables respectively on the minimum and maximum voltage bound. These slack needs to be penalised in the objective function of the DSO's optimisation problems and this cost is added to the shedding cost to form a protections cost.

The complete linear flow model is given by (7). $\forall n \in \mathcal{N}, n^{\prime} \in \mathcal{N}(n), t \in \mathcal{T}, i \in \mathcal{I}$,

$$
\begin{aligned}
& s_{n, t}=\sum_{n^{\prime} \in \mathcal{N}(n)} S_{n, n^{\prime}, t} \\
& S_{n, n^{\prime}, t}=p_{n, n^{\prime}, t}+j q_{n, n^{\prime}, t} \\
& \bar{S}_{n, n^{\prime}, t}=\bar{V}_{0} Y_{n, n^{\prime}}\left(V_{n, t}-V_{n^{\prime}, t}\right) \\
& A_{i} p_{n, n^{\prime}, t}+B_{i} q_{n, n^{\prime}, t} \leq A_{i} C_{n, n^{\prime}} \\
& -A_{i} p_{n, n^{\prime}, t}+B_{i} q_{n, n^{\prime}, t} \leq A_{i} C_{n, n^{\prime}} \\
& A_{i} p_{n, n^{\prime}, t}-B_{i} q_{n, n^{\prime}, t} \leq A_{i} C_{n, n^{\prime}} \\
& -A_{i} p_{n, n^{\prime}, t}-B_{i} q_{n, n^{\prime}, t} \leq A_{i} C_{n, n^{\prime}} \\
& A_{i} e_{n, t}+B_{i} f_{n, t} \leq A_{i}\left(V_{n}^{\text {max }}+\nu_{n, t}\right) \\
& A_{i} e_{n, t}-B_{i} f_{n, t} \leq A_{i}\left(V_{n}^{\text {max }}+\nu_{n, t}\right) \\
& V_{n}^{\text {min }}-\zeta_{n, t} \leq e_{n, t} \\
& \zeta_{n, t} ; \nu_{n, t} \geq 0
\end{aligned}
$$

To run its optimisation problems, the DSO assumes constant power factor in order to estimate injections of reactive power.

\section{TEST SYSTEM AND RESULTS}

\section{A. Test system}

The interaction models are evaluated with the network flow (Netflow) and the linear power flow (LinOPF) model. The test 
system is called Ylpic and originates from a real distribution system that is operated by ORES, a Belgian DSO. It was slightly modified to anonymise sensitive information and to update generation and consumption devices to illustrate what the grid could face in 2020 [9]. This test system contains $328 \mathrm{MV}$ buses in a radial configuration. They are simulated under the same conditions over 12 days, divided in 24 periods and simulated independently. These days are selected to be representative of one year [10], [11], based on the time series of aggregated active and reactive production and consumption of the distribution system. To simulate the tap changer of the $\mathrm{HV} / \mathrm{MV}$ transformer, the voltage at the MV side is considered constant. To some extent, other voltage regulators could help to alleviate voltage violations, but their presence in the test network is very limited. In order to keep the computations tractable, the discrete behaviour of capacitor banks is not optimised. Simulating the twelve days on one interaction model takes respectively 13 and 14 hours of simulation time using the network flow model and the linear power flow model. The experiments are run on a computer equipped with an Intel Core i7 at $3.40 \mathrm{GHz}$ with $32 \mathrm{~GB}$ of RAM, and optimisation problems are solved with SCIP.

To aggregate the large amount of results obtained from simulating a complex system, a simple measure of the welfare is defined as the sum of the benefits and costs of each actor with their sign, and a protection cost. The latter includes the penalty for under and over-voltages as well as the cost of shedding, due to the tripping of a protection (e.g. a selector or a circuit breaker resulting in the complete disconnection of the components behind the protection). This last part adds a notion of quality of service in the welfare, which directly impacts the final consumer of electricity. With this definition, one way to increase the welfare is to increase the amount of renewable generation that is produced within the distribution network, because it directly increases the benefits of the corresponding producers. Moreover, an interaction model, which does not ensure the safe operation of the network, will cause protections to trip and decrease the welfare.

\section{B. Results}

Figure 3 shows the computed welfare of each interaction model, which are all equal when the network flow model is used and hence will not be further analysed in this paper. Integrating voltage constraints highlights the weaknesses of Model 0 and 2, and thus clearly motivates the use of the linear power flow model to assess the performance of interaction models. The detailed contributions to the welfare are given in Table I. The linear power flow model decreases by $20 \%$ the welfare associated with Model 0 . This is directly explained by the same decrease in renewable production. In two of the representative days, congestion of ten lines trigger protections leading to consumption shedding. These two days are problematic in all the interaction models, implying that the flexibility available within the system is not sufficient to solve the issue. Therefore, either more flexibility should be made available, or the network should be reinforced, for example, by increasing the capacity of the ten lines identified by this simulation (in red in Figure 4).

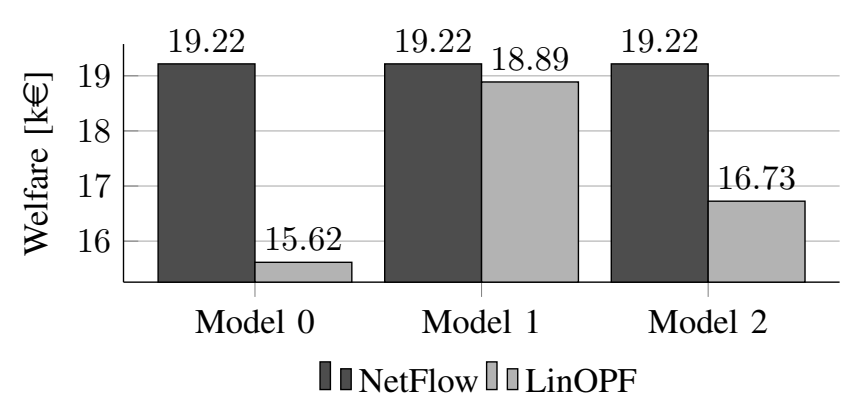

Fig. 3: Welfare of the three interaction models with the network flow model and the linear optimal power flow model.

The welfare increase of Model 1-2 with respect to Model 0 can be first explained by the $26 \%$ increase in the renewable energy production. The welfare of Model 2 is significantly less than the others due to its protection costs, coming from consumption shedding in the fourth representative day during the evening consumption peak. Indeed, Model 2 is based on unrestricted access to the network, and therefore does not force GU to provide flexibility services to the DSO. As a result, too much consumption flows throughout the network and the DSO does not have access to enough flexibility to alleviate the congestion of the lines. Some congestion results from flexibility activation from the TSO within the distribution network. This lack of coordination between the DSO and TSO is solved in Model 1 since grid users were restricted, preventing them from proposing their service to the TSO, see [8] for more details. Previous works have shown that Model 1 outclasses the other tested interaction models using a network flow representation. These results which consider voltage constraints strengthen the latter conclusion.

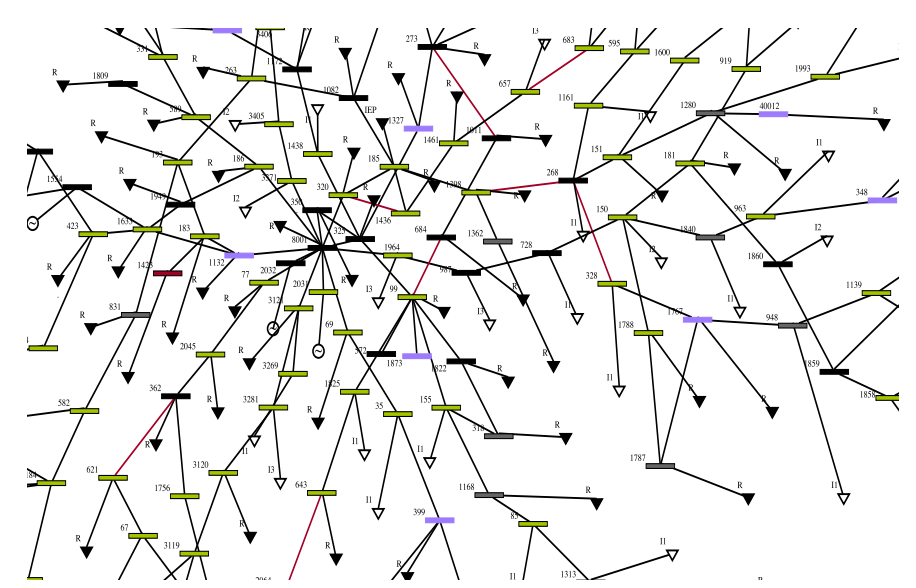

Fig. 4: Summary of the state of one part the Ylpic network for one critical 15-minute period in Model 0. Lines in red are congested. Buses in green contain flexibility activation. The ones in purple suffer under voltages, while the ones in red are shed in real time. 
TABLE I: Impact of the flow model on the interaction models for the Ylpic system.

\begin{tabular}{l|cc|cc|cc|c} 
& \multicolumn{2}{|c}{ Model 0 } & \multicolumn{3}{c}{ Model 1 } & \multicolumn{3}{c}{ Model 2 } \\
& Netflow & LinOPF & Netflow & LinOPF & Netflow & LinOPF & \\
\hline Welfare & 19216 & 15616 & 19216 & 18888 & 19216 & 16725 & $€$ \\
& & & & & & & \\
Protections cost & 0 & 318 & 0 & 283 & 0 & 2393 & $€$ \\
$\begin{array}{l}\text { DSOs costs } \\
\text { TSOs surplus }\end{array}$ & 0 & 0 & 0 & 0 & 0 & 0 & $€$ \\
Producers surplus & 19010.6 & 15891.8 & 19010.6 & 19007.7 & 19010.6 & 19059.7 & $€$ \\
Retailers surplus & 2554.06 & 2534.31 & 2554.06 & 2535.32 & 2554.06 & 2407.57 & $€$ \\
& & & & & & & \\
Total production & 633.14 & 501.1 & 633.14 & 624.22 & 633.14 & 629.05 & MWh \\
Total consumption & -1142.74 & -1141.4 & -1142.74 & -1141.53 & -1142.74 & -1133.44 & MWh \\
& & & & & & & \\
Total imbalance & 0 & 0.32 & 0 & 0.28 & 0 & 1.58 & MWh \\
Max. imbalance & 0 & 0.03 & 0 & 0.03 & 0 & 0.29 & MW \\
Total usage of flex. & 29.63 & 26.82 & 29.63 & 27.42 & 29.63 & 29.63 & MWh \\
Total energy shed & 0 & -0.32 & 0 & -0.28 & 0 & -1.3 & MWh
\end{tabular}

\section{CONCLUSION}

This paper extends the DSIMA testbed with a linear approximation of the power flows, integrating reactive powers and voltage constraints. It is based on Bolognani and Zampieri's work which provides a linear relation between the voltages and the injected power at each bus [4]. This linear flow model is compared to the network flow model by simulating three different interaction models governing the exchange of flexibility services within a realistic distribution system. Results show that changing the network does impact the quantitative results obtained from the simulations when voltage constraint are binding. Indeed, using a network flow model provides similar welfares, whereas using a linear power flow model allows one to decide which interaction model to choose. The simulations suggest that the operation of the tested distribution network should rely on dynamic access ranges.

This work can be extended along several lines. One could refine the modelling of the reactive power injections by developing a dedicated prediction for the DSO and add uncertainty to these injections in its optimisation problems. The exchange of reactive power flexibility services would be worth implementing to assess its impact on the network operation costs. Finally, new interaction models that target the reactive power support could be quantitatively assessed.

\section{ACKNOWLEDGMENTS}

This research was supported by the public service of Wallonia - Department of Energy and Sustainable Building, within the framework of the GREDOR project.

\section{REFERENCES}

[1] Q. Gemine, E. Karangelos, D. Ernst, and B. Cornélusse, "Active network management: Planning under uncertainty for exploiting load modulation," in Bulk Power System Dynamics and Control-IX Optimization, Security and Control of the Emerging Power Grid (IREP), 2013 IREP Symposium, IEEE, 2013, pp. 1-9.

[2] L. Tesfatsion and K. L. Judd, Handbook of computational economics: agent-based computational economics. Elsevier, 2006, vol. 2.
[3] S. Mathieu, Q. Louveaux, D. Ernst, and B. Cornélusse, "Dsima: A testbed for the quantitative analysis of interaction models within distribution networks," Sustainable Energy, Grids and Networks, vol. 5, pp. 78-93, 2016.

[4] S. Bolognani and S. Zampieri, "On the existence and linear approximation of the power flow solution in power distribution networks," Power Systems, IEEE Transactions on, no. 99, 2015, ISSN: 0885-8950.

[5] B. Stott, J. Jardim, and O. Alsaç, "DC power flow revisited," Power Systems, IEEE Transactions on, vol. 24, no. 3, pp. 1290-1300, 2009.

[6] A. Farag, S. Al-Baiyat, and T. Cheng, "Economic load dispatch multiobjective optimization procedures using linear programming techniques," Power Systems, IEEE Transactions on, vol. 10, no. 2, pp. 731-738, 1995.

[7] C. Coffrin and P. Van Hentenryck, "A linearprogramming approximation of ac power flows," INFORMS Journal on Computing, vol. 26, no. 4, pp. 718734, 2014.

[8] S. Mathieu, D. Ernst, and B. Cornélusse, "Agent-based analysis of dynamic access ranges to the distribution network," in Innovative Smart Grid Technologies Europe (ISGT EUROPE), 2016 6th IEEE/PES, IEEE, 2016.

[9] B. Cornélusse, D. Vangulick, M. Glavic, and D. Ernst, "A process to address electricity distribution sector challenges: The GREDOR project approach," in Proceedings of the International Conference on Electricity Distribution, CIRED 2015, 2015.

[10] S. Mathieu, DaysXtractor. [Online]. Available: https: //github.com/sebMathieu/daysxtractor.

[11] K. Poncelet, H. Hoschle, E. Delarue, A. Virag, and W. D'haeseleer, "Selecting representative days for capturing the implications of integrating intermittent renewables in generation expansion planning problems," IEEE Transactions on Power Systems, 2016. 